\title{
Comparison of Mutual Information with a Standard Method for Alignment of Histological Serial Sections
}

\author{
Ying $\mathrm{Yu}$ \\ Department of Biomedical Engineering \\ China Medical University \\ Shenyang, China
}

\author{
Joergen Erik Assentoft \\ Department of Nuclear Medicine \& Department of Medical \\ Physics \\ Aalborg University Hospital \\ Aalborg, Denmark
}

\author{
Jesper Skovhus Thomsen, Erik Ilsø Christensen, Arne Andreasen \\ Institute of Anatomy \\ Aarhus University \\ Aarhus, Denmark
}

\begin{abstract}
The purpose of the study was to compare the ability of a mutual information algorithm with that of a standard algorithm to align images of histological serial sections. The two align algorithms were implemented in $\mathrm{C}$ running on a Linux based PC. Both algorithms used the same gradient-based optimizer, but different cost functions standard (ST), and mutual information (MI) respectively. The object of the test was to align 4557 serial sections originating from a rat kidney. The alignment of kidney sections is difficult as these sections contain many nearly identical tubules, representing a high degree of translation symmetry. As a consequence there is a non-negligible chance of misalignment into a local minimum, making serial kidney sections good real life test objects for image alignment. We showed that images, which were difficult to align by the $S T$ were easy to align with $M I$. We found that the most efficient strategy was first to align all 4557 images using the $S T$ function and then to align the misaligned 54 images using the $M I$ function.
\end{abstract}

Keywords: Alignment; mutual information; PET; registration; serial sections; transformation values.

\section{INTRODUCTION}

$3 \mathrm{D}$ reconstruction from consecutive histological sections has proved a useful tool. When an object is inspected in a given section, it can later on be inspected in any anatomical plane in space, and new biological information can be revealed. This has proven useful in the study of the microstructure of the mouse kidney [1,2], where multiple tubules (nephrons) were meticulously traced several times back and forth through more than 5400 serial sections. However, a high quality of the alignment of the histological section is crucial if such tracings are to be feasible. Moreover, the alignment of the histological sections must be achieved inside a reasonably time frame. Thus, the speed of the alignment program is one of the key parameters, important for the day-to-day procedures.
During alignment of two images one image is translated and rotated relative to the other (reference) image and the two images are compared with a function, which quantifies how well the two images correspond. Such a function is referred to as a cost function. Therefore, the object of the image aligning procedure is to minimize the cost function. Another function, called the optimizer, is applied in repeated cycles is controlling the relative translations and rotation of the images so as to minimize the cost function $[3,4]$. If the two images are not identical but closely related, as e.g. two adjacent images, the minimal cost function value will be larger than zero, but have a global minimum at the best fit position. In this way, the cost function can be used as a parameter for the alignment. With the images roughly aligned, the alignment program calculates an estimate for the relative translation $d x$, then $d y$ and finally the rotation $d \theta$ around a point $x, y$ during a set of repeated cycles, until convergence [5-7]. The simplest cost function compares the intensity of all corresponding pixels in the two images $\left(S T=\sum\left|I_{1}-I_{2}\right|\right)$. However, even though this simple sum function is fast to determine, it is calculated many times during each cycle, which cost time. When aligning histological kidney sections, we have found that approximately $1 \%$ of the aligning attempts end up in a local minimum rather than in the desired global minimum. Such misaligned images or "outs" must be realigned with another set of initial conditions, which requires additional computation time. Instead of "carpet bombing" the initial condition parameter space with a series of different initial conditions in order to find the global minimum of the cost function, another more time consuming cost function could be applied. Depending on the purpose and nature of the aligned image material, various alternative and more complex cost functions have been used [4].

The mutual information (MI) function has been widely used for alignment (registration) of PET and CT images [8]. In 
this context, $M I$ is just a function, based on a common or mutual histogram, i.e. a matrix suspended by the pixel values in the two images under alignment. Alignment by $M I$ is in general considered less sensitive to local optima [3], but it is also more time consuming, as $M I$ is a rather complex function. Therefore, the aim of the study was to test $M I$ versus the simple $S T$ function for the alignment of serial histological sections, concerning speed and quality. The test material consisted of histological serial sections of the rat kidney that contains multiple tubules, resulting in a high degree of translation symmetry, making the alignment of these images non-trivial.

\section{MATERIALS AND METHODS}

\section{A. Preparation}

The kidney from a 3-months-old Wistar rat was fixed by retrograde perfusion through the abdominal aorta with $1 \%$ glutaraldehyde, embedded in Epon, cut into $45572.5-\mu \mathrm{m}-$ thick serial sections, and stained with toluidine blue.

\section{B. Image Recordings}

The sections were digitized using an Olympus AX 70 microscope equipped with a digital camera (Olympus DP 50; Olympus, Tokyo, Japan). Up to $3 \times 3$ overlapping digital recordings from each histological section were combined into one 24-bit color image by use of "analySIS" (Soft Imaging System, Version 3.2; Soft Imaging System, Münster, Germany). The images were given a common $6000 \times 6000$ pixel canvas. The magnification corresponded to a pixel size of $1.52 \mu \mathrm{m}$. Finally, a copy of the images was converted to grey-scale images for aligning purposes.

\section{The Alignment Program}

The aligning procedures were implemented in a computer program written in $\mathrm{C}$ running on Linux (OpenSUSE 11.3). Both aligning procedures used the same gradient-based optimizer, whereas the cost function (ST or $M I$ ) was a compile time option. Both aligning procedures are intrinsic, i.e. they work without fiducial markers. With the images roughly aligned, the alignment program can calculate an estimate for the relative translation $d x$, then $d y$ and finally the relative rotation $d \theta$ around a point $x, y$, during a set of repeated cycles until convergence. The ST is given by:

$$
S T=\sum_{1}^{n}\left|I_{1_{i}}-I_{2_{i}}\right|
$$

whereas the $M I$ is given by $[3,4]$ :

$$
M I=I(A, B)=\sum_{a, b} \log _{2} \frac{p(a, b)}{p(a) \times p(b)}
$$

A detailed description of the $M I$-operator was given by Viola and Wells [3]. For practical reasons, the output from the $M I$ was inverted so that the optimizer should search for a minimum regardless of whether working with the $S T$ or the $M I$ function.

\section{Control of the alignment based on $S T$}

Relative transformation values obtained by the $S T$ were added into a set of absolute transformation values. The absolute transformation values underwent a high-pass filtration in order to avoid potential distortions of the image stacks as caused by small but accumulating trends [5-7]. The original 4557 color images were then transformed according to the filtered absolute transformation values. For quality control of the alignment, the alignment program made the two images into an alignment pair semitransparent and fused them onto each other. These fused images were inspected one by one using a standard image viewing program (F-Spot Photo Viewer) on the Linux platform.

In another control test the 4557 transformed images were fused into $4557-1$ couples and shown as animated films.

\section{E. Selection of test images}

The control showed that 54 of the 4557 alignments based on the $S T$ function, were "outs". Five of these images, and five representative control images, which had been well aligned, were selected for extensive alignment tests by both $S T$ and $M I$. In the following, we denote these five "outs" as "problems" and the five with a good alignment as "controls".

\section{F. Time measurements}

One representative couple of images was selected for 5 types of pixel reduced modes:

Firstly (step 1), the $S T$ and $M I$ were calculated with all pixel values in the two images taken into account. Secondly (step 2), the $S T$ and $M I$ were calculated for only every second corresponding value in the $x$ - and $y$-direction, i.e. only quarter of the pixel values were included. Then, in step 4 every $16^{\text {th }}$, in step 8 every $64^{\text {th }}$, and in step 16 every $256^{\text {th }}$ pixel value were included.

\section{G. Quality measurement of alignment by ST versus $M I$}

I order to estimate the error frequency, each image pair was aligned 1000 times with a new initial condition each time. The alignments were performed by $S T$ and $M I$ on the 5 controls and the 5 problem image pairs at step 1, 2, 4, 8, and 16. This resulted in a total of $1000 \times 2 \times 2 \times 5 \times 5=100,000$ relative transformation values, listed in a text file. The five controls and the five problem images were transformed according to the 100,000 relative transformations, and fused in pairs to their respective reference image. For each of the fused images the local contrast in the $3 \times 3$ neighbourhood around a pixel was calculated as $C=\left(I_{\max }-I_{\min }\right) /\left(I_{\max }+I_{\min }\right)$. Then a contrast index $C I$ was determined as the mean of all the $C$ values in the image. The 100,000 fused image pairs were listed 


\section{computation time}

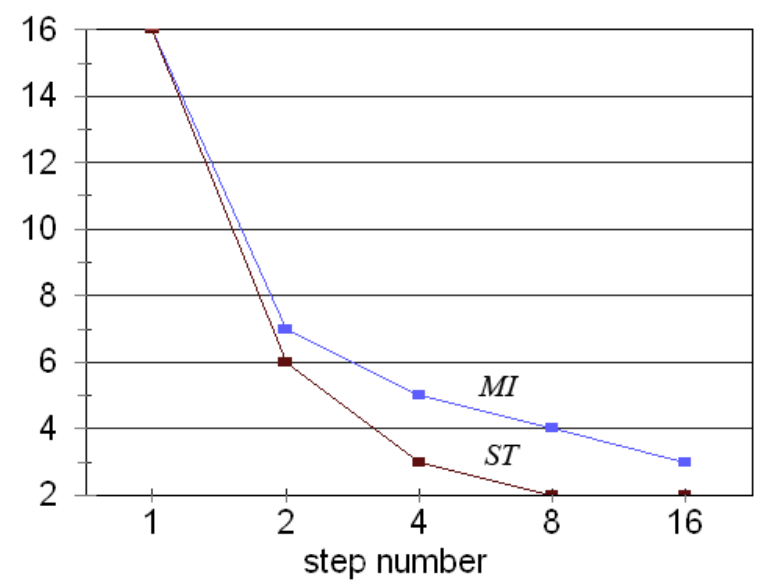

Figure 1. Average computation time per alignment for $M I$ (blue line) and $S T$ (red line) as a function of step number.

according to their $C I$ value and inspected in the image viewing program. The grid size was determined by the diameter of the tubules, and optimized by a test sweep.

\section{RESULTS}

Time consumption as function of step number for $S T$ and $M I$ is shown in figure 1.

The speed of the $S T$ and the $M I$ was similar in step 1 and step 2, whereas at step 8 , the $S T$ was twice as fast as the $M I$. When the 100,000 fused image pairs were inspected in the image viewing program it turned out that well aligned image pairs had the highest $C I$, whereas the "outs" had the lowest $C I$. The error frequencies i.e. the frequencies of "outs" are summarized in figure 2 and 3.

The $M I$ had an error frequency that was as good as or better than the $S T$ when dealing with the problem images. The

\section{problems}

MI versus-ST

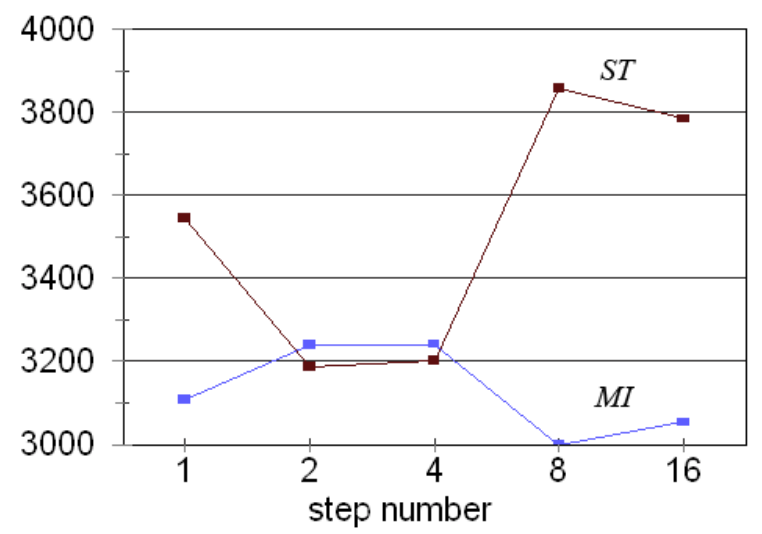

Figure 2. Number of "outs" per 25,000 alignments of problem images for $M I$ (blue line) and $S T$ (red line).

\section{controls}

MI versus-ST

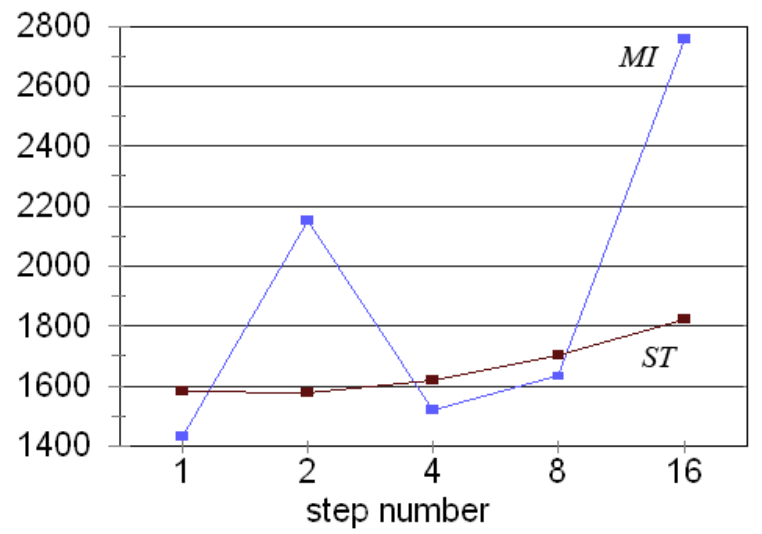

Figure 3. Number of "outs" per 25,000 alignments of control images for $M I$ (blue line) and $S T$ (red line).

lowest error frequency occurred at step 8 with 3000 "outs" of 25,000 or $12 \%$, (figure 2). From visual inspection it seemed that the problem images had a high density of similar appearing tubules, and that the $M I$ was more resistant than the $S T$ to this translation symmetry. In contrast, the $S T$ seemed in general to have a lower error frequency than the $M I$ when dealing with the control images, although the $M I$ had a lower error frequency than the $S T$ at step 4 and 8 .

In conclusion, the most efficient strategy seems to be to align all images by the $S T$ at step 8 , and then to realign the "outs" by $M I$, also at step 8 .

The $C I$ guided judgment of the 100,000 fused image pairs showed that one alignment could have different solutions where a larger or smaller part of the two images are matching. In most cases, such different solutions are local minima, sometimes reflecting translation symmetry, rotation symmetry, or a combination of both, as shown in figure 4. Most important, these different solutions were clearly discriminated by the simple contrast index $C I$. In other cases, there could be two different, but just as good solutions, with nearly the same contrast value. This was always caused by artifacts, where the tissue section had a fold leaving two nearly equal areas. The alignment program always selected just one of the areas, instead of something in between. The cases with two or more nearly equal good solutions caused by artificial folds emphasize the importance having high quality histological sections.

\section{DISCUSSION}

The purpose of the study was to test the $M I$ against the simple $S T$ function for the alignment of serial histological sections, concerning speed and quality.

In the actual context the term mutual information has been regarded in its restricted meaning linked to the mutual histogram. In a wider context, the term also emphasize that the alignment/registration can take different qualities of 


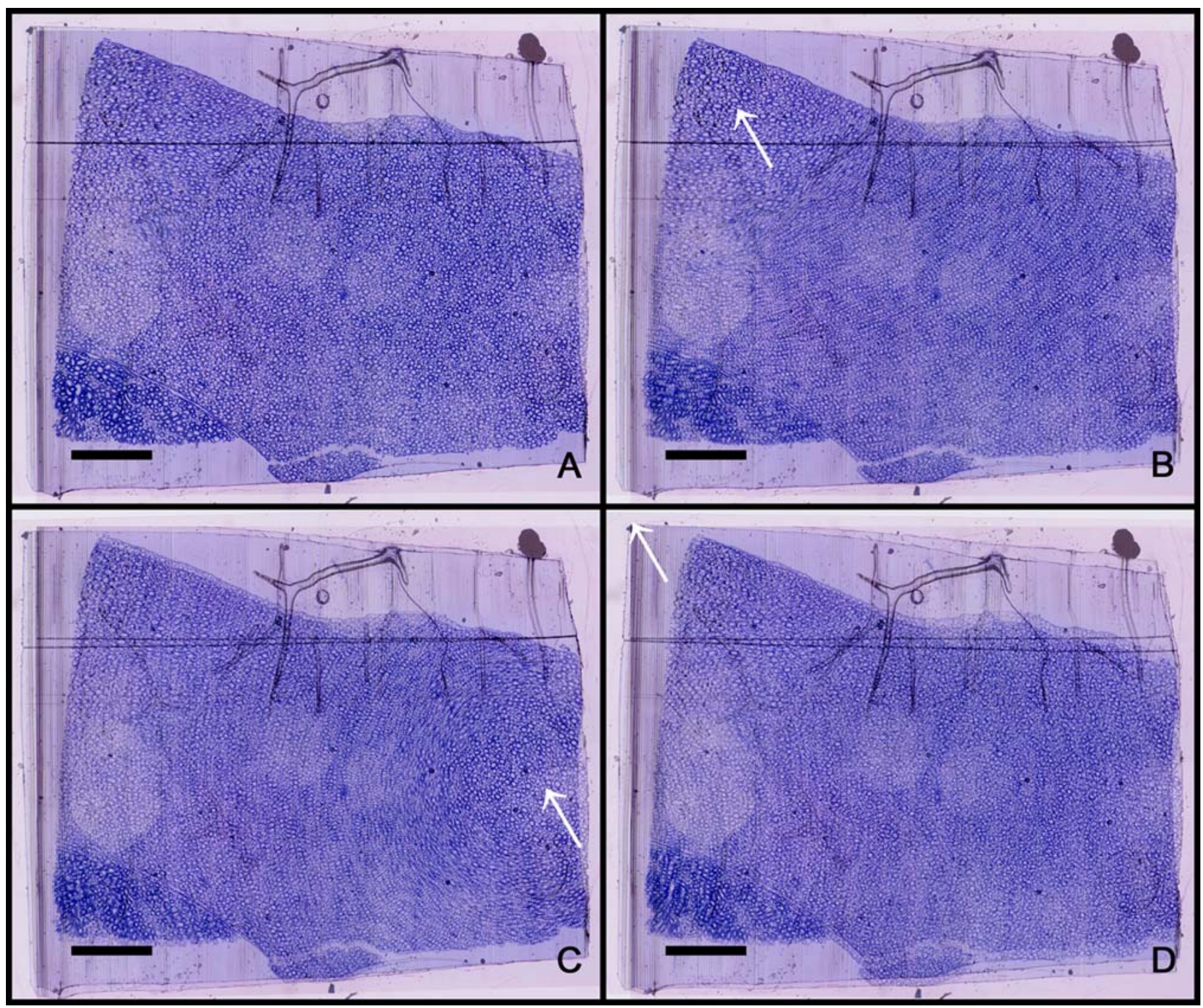

Figure 4. Four of 1000 fused image couples in one of the 100 tests. The images are arranged with the decreasing contrast index $C I$ (a high contrast index corresponds to a good fit). A has a global optimum with the most high $C I=14.6527$. B local minimum with match in the upper left corner (arrow), $C I=13.0551$. $\mathrm{C}$ local minimum with match around a point in the middle to the right (arrow), $C I=13.0083$. D local minimum with match only in the upper left corner (arrow), $C I=12.9893$. The horizontal line in the upper part is an artificial marker from the cutting procedure, left by the microtome knife. The simple contrast index was an efficient selector of good alignments and "outs". Bar $=1 \mathrm{~mm}$, tubular diameter 5-70 $\mu \mathrm{m}$.

information into account, e.g. color, edges, contrast, form, or other things. This means that a simple but specific alignment/registration may require a specific kind of preprocessing for enhancement of the relevant quality [8,9].

In this study, with the actual kidney sections, there was no need for any further pre-processing or image enhancement. The reason for this was the high quality of the histological sections, and the excellent toluidine blue staining, as well as the pre-settings of the microscope.

The alignment of the kidney sections is difficult because the sections contain a network of multiple, similarly appearing tubules, resembling chicken wire, representing a high degree of translation and rotation symmetry. We regard the alignment of kidney sections as a worst case scenario, making kidney sections the best non-artificial test object.

We found the most efficient strategy first to align all images by the $S T$ at step 8, and then to realign the "outs" by $M I$ at step 8. This knowledge can save a lot of time.

Concerning the control images, the $M I$ had a low error frequency at step 1, then a higher at step 2, and again a low frequency at step 4. This result suggests that a reduction in information can in some cases make the local minimum more deep, while in other cases can make the local minimum less deep. A similar phenomenon was not seen with the $S T$ procedure. This result suggests the need of a step test sweep before running many $M I$ realignments. 
Most importantly, the result implies that the $M I$ can be useful, not only for registration of PET/CT images, but also for the alignment of histological serial sections.

Despite the fact that our time measurement are specific for the actual histological and material and setup, it may be a general result that the $M I$ is more efficient than the $S T$ for images with high translation symmetry and multiple potential local optima.

The $M I$ is more complex and therefore slower than the $S T$. The MI matrix is suspended by the bit depth, here 8 bits, resulting in a $256 \times 256$ matrix. Another way to reduce computation time is to reduce the bit depth of the considered images thereby reducing the size of the $M I$ matrix. It is conceivable that a bit depth of e.g. 6 , and thereby a MI matrix of $64 \times 64$ ), would contain enough information to successfully align the images under consideration. It is worth noting that this will not affect the quality of the outcome, because the final absolute transformation can be performed using images of the original bit depth. However, a formal investigation into this is outside the scope of the present study.

A potential general speeding up process outside the $M I$ versus $S T$ context has recently become easier with the access to hardware acceleration by means of the Graphic Processor Unit (GPU), [10, 11]. The idea is that the program can look for, and utilize vacant potential calculating hardware in the given computer configuration. This means that $M I$ can work fast itself, without the fast initial alignment with $S T$.

The contrast index $C I$ proved efficient in selecting the different groups of solutions among the 1000 fused image pairs in the 100 tests. Therefore, we propose $C I$ as a potential alternative to the fast $S T$, i.e. that $C I$ can work as a fast cost function itself.

\section{ACKNOWLEDGMENT}

The authors are grateful for the excellent technical assistance of Inger B. Kristoffersen. We also want to thank Birgitte Rasmussen and Birgitte Lundbøl Grann for linguistic revisions of the manuscript. We want to gratefully acknowledge Bodil Marie Lousdal, Lotte Nyholm Andersen, Jonna Lisberg, and Dorthe Askholm, Aalborg University Hospital, for much practical help and support. We would like to thank Ivan Noer and Jesper Carl, Department of Nuclear Medicine \& PET/CT and Department of Medical Physics, Aalborg University Hospital, for financial support and help.

Thank to Ole Fejerskov, Institute of Anatomy for support and Kristian Thorn, University of Aarhus, for dealing with The Danish immigration Service. Finally, we would like to thank Jens Møller Jensen and Sitaraman Arun Krishna, Danfoss IXA, for much support.

\section{REFERENCES}

[1] X-Y. Zhai, H. Birn, K.B. Jensen, J.S. Thomsen, A. Andreasen, and E.I. Christensen, "Digital three-dimensional reconstruction and ultrastructure of the mouse proximal tubule", J. Am. Soc. Nephrol., vol. 14, pp. 611$619,2003$.
[2] X-Y. Zhai, JS. Thomsen, H. Birn, IB. Kristoffersen, A. Andreasen, and EI. Christensen, "Three dimensional reconstruction of the mouse nephron", J. Am. Soc. Nephrol., vol. 17, pp. 77-88, 2005.

[3] P. Viola and W.M. Wells III, "Alignment by maximization of mutual information”, Int. J. Comput. Vis, vol. 24, pp. 137-154, 1997.

[4] R. Shams, P. Sadeghi, R.A. Kennedy, and R.I. Hartley, "A survey of medical image registration on multicore and the GPU", IEEE Signal Process. Mag., vol. 27, pp. 50-60, 2010.

[5] A. Andreasen, A.M. Drewes, J.E. Assentoft, and N.E. Larsen, "Computer-assisted alignment of standard serial sections without artificial landmarks. A practical approach to the utilization of incomplete information in 3-D reconstruction of the hippocampal region", J. Neurosci. Methods, vol. 45, pp. 199-207, 1992.

[6] A. Andreasen, G. Danscher, S. Juhl, N.P. Revsbech, H. Jensen, and K.B. Jensen, "Distinct differences in partial oxygen pressure at micrometer ranges in the rat hippocampal region", J. Neurosci. Methods, vol. 72, pp. $15-21,1997$.

[7] M. Stoltenberg, A. Andreasen, K.B. Jensen, S. Juhl, G. Danscher, and E. Ernst, "PC-assisted three-dimensional description of organs containing tubular structures. Applied on the epididymis of the rat", Comput. Med. Imag. Grap., vol. 21, pp. 323-329, 1998.

[8] J.E. Assentoft, J.S. Thomsen, and A. Andreasen, "Super-sampling in PET. A concept for improvement of the resolution of positron emission tomography", Conf. Proc. IEEE Eng. Med. Biol. Soc., vol 1, 3622-3625, 2010.

[9] X-Y. Zhai, E.I. Christensen, and A. Andreasen, "Low noise electron microscopy by merging multible images digitized from conventional films with reference to the mouse kidney", Microsc. Microanal., vol. 12, $1-7,2006$.

[10] R. Shams and N. Barnes, "Speeding up mutual informa-tion computation using NVIDIA CUDA hardware", Proc. Digital Image Computing: Techniques and Applications (DICTA), Adelaide, Australia, pp. 555560, 2007.

[11] R. Shams, P. Sadeghi, R.A. Kennedy, and R. Hartley, "Parallel computation of mutual information on the GPU with application to realtime registration of 3D medical images", Comput. Methods Programs Biomed., vol. 99, pp. 133-146, 2010. 